\title{
Static Metasurface Reflectors with Independent Magnitude and Phase Control using Coupled Resonator Configuration
}

\author{
Joel S. Demetre Student Member, IEEE, Tom. J. Smy, and Shulabh Gupta Senior Member, IEEE
}

\begin{abstract}
A static metasurface reflector based on a novel coupled resonator configuration is proposed to independently control the reflection phase and magnitude of linearly polarized incident fields, and is demonstrated experimentally in the millimeter-wave Ka-band around $30 \mathrm{GHz}$. The proposed concept is illustrated using a unit cell design consisting of a rectangular ring coupled with a rectangular slot resonator backed by a grounded dielectric slab. By geometrically tuning various dimensions of the two resonators, a near-perfect amplitude-phase coverage is achieved at a fixed design frequency of $30 \mathrm{GHz}$. To demonstrate the flexible beam-forming capability of the proposed metasurface reflectors, illustrative examples of fixed beam steering with varying reflection magnitudes, and asymmetric dual-beam patterns with specified reflection magnitude, reflection angles and beam-widths, are successfully shown. Compared to the standard method based on polarization rotation and resistive loadings with discrete values, the proposed technique does not generate undesired crosspolarization field reflection, and provides a continuous magnitude tuning including full absorption, along with wide phase coverage.
\end{abstract}

Index Terms-Electromagnetic Metasurfaces, Metasurface Reflectors, Coupled Resonators, Complex Reflectance, Independent Amplitude and Phase Control, Far-field Beam-forming.

\section{INTRODUCTION}

Electromagnetic metasurfaces are 2D arrays of subwavelength resonating particles, which are engineered at the microscopic scale to tailor the macroscopic scattered fields in response to the specified incident fields. By manipulating their sizes, materials and geometrical shapes, wide variety of wave transformations in transmission and reflection, along with polarization control, can be achieved [1], [2]. Consequently, they have found a myriad of applications ranging across the electromagnetic spectrum, from radio frequencies (RF) to optical [3]-[10].

To achieve complete control over the scattered fields from a metasurface, the sub-wavelength resonating particles must be engineered to provide all possible combinations of transmission/reflection phase and magnitude. If the surface is reflection-type, only the reflection magnitude and phase must be controlled, i.e. the complex reflectance. However, if the surface is transmission-type, the problem is more involved, as both complex transmittance and reflectance must be simultaneously manipulated. Once such a resonating particle is conceptualized and designed, the resulting complex reflectance (and/or transmittance) can then form the building blocks to

J. S. Demetre, Tom J. Smy, and Shulabh Gupta are with Carleton University, Ottawa, Canada (e-mail: JoelDemetre@cmail.carleton.ca). realize an arbitrary spatial distribution of reflection field profile to obtain desired scattered fields.

Few solutions to achieve an independent reflection magnitude and phase have been attempted in the literature. These existing solutions can fundamentally be divided into two categories: 1) Polarization rotation, and 2) Resistive/Lumped element loading. In the first approach, part of the incoming polarization is converted into a cross-polarized field component, which acts as an effective loss or amplitude modulation of the specified fields. The geometrical shape of the particle is typically used to independently tune the phase [11]-[14]. This strategy can be applied in both reflection and transmission operation [15]-[20]. While this is an effective method to control the amplitude and phase, it naturally generates spurious cross-polarized fields, which either must be ignored or be selectively absorbed.

The second approach is to use resistive loadings in the resonators to modulate its Q-factor. This is typically achieved by adding lumped resistive components in strategic locations in the unit cell to change the magnitude, while the phase is tuned using the geometrical dimensions or by lumped inductors and capacitors [21]-[24]. For static cases, these components are typically off-the-shelf commercially available RF resistors, for magnitude control. For dynamic cases, electronically tunable elements such as varactor diodes and PIN diodes are naturally required to modulate the phase and amplitude independently, as as recently been shown for metasurface reflectors [25], [26]. In these approaches, no cross polarization is generated, as desired. For static surfaces, since the amplitude control is through RF resistors, which are typically available in discrete values only, there is no simple way to achieve a continuous range of resistance values (and thus the magnitude values). Moreover, from the fabrication perspective, it requires an extra lumped element assembly step, which increases its prototyping costs. The use of lumped elements also presents inherent difficulties when scaling to higher frequencies or when moving to a more sub-wavelength unit cell; as these elements have a fixed size in comparison to the unit cell.

In this work, we are proposing a third method of achieving independent reflection magnitude and phase using a static metasurface reflector, when operated with linearly polarized incident waves with zero cross-polarization, and which does not rely on discrete static lumped resistors. This proposed technique is based on a concept inspired by [25], where the metasurface cell consists of two tightly coupled resonators. Dimensional changes in one resonator affects the reflection re- 

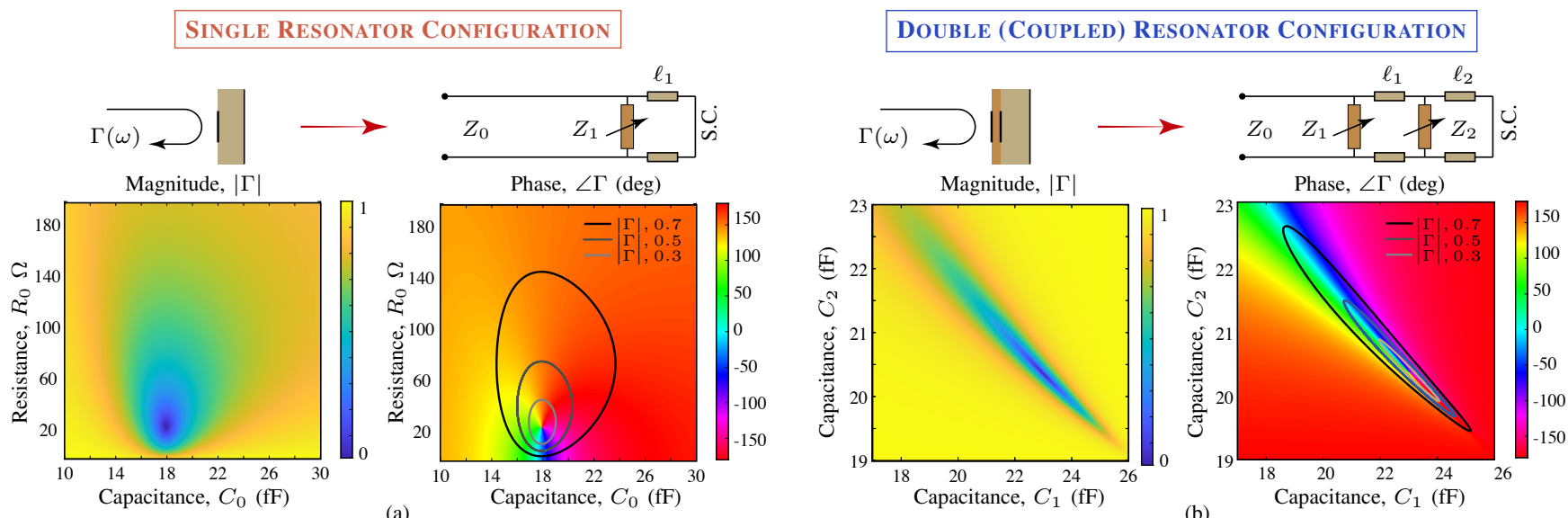

Fig. 1. A comparison between a conventional single resonator unit cell configuration and the proposed dual (coupled) resonator configuration, using an equivalent transmission line model where each resonator is modeled as an LCR circuit. a) Amplitude and phase coverage as a function of capacitance, $C_{0}$ and resistance $R_{0}$, showing contours of constant magnitude, computed using Advanced Design System (ADS) circuit modeler and validated with an ABCD matrix cascade approach [27]. b) Amplitude and phase coverage as a function of the two capacitances, $C_{1}$ and $C_{2}$, showing contours of constant magnitude. Simulation parameters: a) $L_{1}=1.06 \mathrm{nH}, \ell_{1}=200 \mu \mathrm{m}, \epsilon_{r}=3.66, \delta=0.004$, b) Fixed resistance $0.9 \Omega$ and inductances $1.06 \mathrm{nH}, \ell_{1}=200 \mu \mathrm{m}$, $\ell_{2}=750 \mu \mathrm{m}, \epsilon_{r}=3.66, \delta=0.004$.

sponse of the second resonator, via electromagnetic coupling, enabling an efficient way to independently, and continuously, tune the reflection amplitude and phase. The proposed concept is illustrated using both transmission line models and full-wave simulations, and is experimentally confirmed using variety of reflector prototypes to produce steered beams with varying amplitude and asymmetric dual beam profiles.

The paper is structured as follows. Sec. II presents the comparison of a single resonator surface with a double resonator using a transmission line model and introduces the concept. Sec. III shows the proposed unit cell architecture, and demonstrates independent amplitude and phase tuning using Ansys FEM-HFSS, along with proposed practical metasurface implementation. Sec. IV presents the fabricated metasurface reflector prototypes for various wave transformations in the Ka-band around $30 \mathrm{GHz}$, and the custom build metasurface measurement system used to characterize the surfaces. Conclusions are finally provided in Sec. V.

\section{Proposed Metasurface Principle}

\section{A. Conventional Single Resonator Unit Cell}

A conventional reflective metasurface unit cell typically consists of a sub-wavelength resonator on top of a host dielectric backed by a ground plane, as illustrated in Fig. 1(a). To understand its reflection characteristics, an equivalent transmission line model may be built [28]-[30], where a generic resonator is represented by a shunt resonator, $Z_{1}$, which could be a series $L C R$ resonator, for instance, i.e.

$$
Z_{1}(\omega)=\left(R_{0}+j \omega L_{0}+\frac{1}{j \omega C_{0}}\right)
$$

It is followed by a transmission line of length $\ell$, corresponding to a dielectric slab of permittivity $\epsilon_{r}$, backed by a short circuit representing the ground plane. A resistance is added to account for the inherent conductor losses.

Fig. 1(a) shows a typical reflection magnitude and phase response, as the resistance and the capacitance of the resonator is changed. While the capacitance change may be brought about by changing the dimension of the resonator geometry, to tune its resonant frequency, a precise change of the resistance is only possible by adding external lumped elements. The amplitude and phase plots reveal, that an effective tuning of the phase is achieved as capacitance is changed while keeping a constant resistance, $R_{0}$. However, this is accompanied by an uncontrolled change in the magnitude. The figure further shows various contours of constant magnitudes, which offers full $2 \pi$ phase, however it confirms that an independent magnitude and phase control in this single resonator configuration, necessarily requires simultaneous tuning of capacitance and resistance [21].

\section{B. Proposed Coupled-Resonator Unit Cell}

It is clear from the example of a single resonator of Fig. 1(a), that two control elements are needed to independently tune the reflection magnitude and phase. To avoid resistance tuning, an alternative way is to add another resonator structure, resulting in a dual resonator configuration, as illustrated in Fig. 1(b). The resonance control of each of the two resonators thus can be seen as two independent tuning mechanisms to manipulate its complex reflectance. This is more clearly seen in an equivalent transmission line model, where the two resonators are chosen to be separated by a dielectric slab, which are both placed on a short-circuited host dielectric. Each of the resonators are considered here to be a series $L C R$ resonator, where their capacitances $C_{1}$ and $C_{2}$ maybe varied to tune their resonance frequencies. Such resonance shifts can be practically brought about by structural changes in the two resonators. While the two resonators in this equivalent model are shown as uncoupled for simplicity, in physical practical implementations, they will naturally be electromagnetically coupled.

The typical reflection amplitude and phase response is further shown in Fig. 1(b) for varying values of the two capacitances, $C_{1}$ and $C_{2}$, and for a fixed value of the resistance. The reflection features are distinctly different from the case of a single resonator, while still containing regions 
of full reflection and absorption, along full phase coverage. To evaluate if an independent tuning of reflection phase and magnitude is possible, contours of constant magnitude are shown in the phase plots for various values. It is clearly visible that each of these constant magnitude contours pass through the full range of reflection phase. In other words, specific combinations of $C_{1}$ and $C_{2}$ are possible to achieve a specified arbitrary combination of reflection magnitude and phase at the desired frequency, without changing the resistance. This thus provides an opportunity for complete control over the complex reflectance by tuning the resonant frequencies of each resonator, which can be easily accomplished by changing their geometrical dimensions and without adding any extra lumped circuit elements, as will be demonstrated next.

\section{Metasurface ReFlector Design}

\section{A. Proposed Unit Cell Design}

The proposed metasurface reflector of Fig. 1(b) requires a sub-wavelength double resonator configuration backed by a grounded dielectric slab. A simple practical implementation of such a configuration is shown in Fig. 2(a) chosen here for a $30 \mathrm{GHz}$ operation in the Ka-band, for later demonstration. It consists of a rectangular ring resonator coupled with a rectangular slot resonator, which is then placed on top of a conductor backed dielectric slab. Any changes in one resonator affects the response of the second resonator via electromagnetic coupling, which is then used to achieve independent amplitude-phase tuning. The resulting unit cell has a sub-wavelength periodicity i.e. $\Lambda \ll \lambda_{0}$ (e.g. $2 \mathrm{~mm}$ for this example equals $\lambda_{0} / 5$ at $30 \mathrm{GHz}$ ). Practically, the proposed unit cell architecture is a multi-layer configuration, as shown in Fig. 2(a), where the two dielectric layers are attached using a bonding layer. The unit cell layer stack has a ground plane of thickness $52 \mu \mathrm{m}$, rectangular slot of $35 \mu \mathrm{m}$ and rectangular ring $35 \mu \mathrm{m}$, all being made of copper. The base substrate $\left(h_{1}=730 \mu \mathrm{m}\right)$ and the top substrate $\left(h_{2}=100 \mu \mathrm{m}\right)$ are both composed of Rogers 4350B, which was simulated using an $\epsilon_{r}$ of 3.62 and a $\delta$ of 0.0037 . The PrePreg bonding layer $(t=110 \mu \mathrm{m})$ was made of Rogers RO4450F and simulated using an $\epsilon_{r}$ of 3.8 with a $\delta$ of 0.0039 . Nine micron thick resin layers were used adjoining the metal and substrate/PrePreg which have an $\epsilon_{r}$ of 2.4. These design parameters are used throughout this work for later prototyping and testing.

The unit cell operates on a linearly polarized incident waves. Due to the symmetry of the structure, no cross-polarization is generated, as desired. If the slot resonator has zero width, the proposed cell simply becomes a conventional single resonator structure with a uniform ground plane. The addition of a slot in that ground, can thus be seen as introducing an effective defected ground plane structure, with engineerable surface impedance, instead of a fixed short-circuit impedance [31]. While the proposed unit cell structure with slot resonator maybe seen as a simple and intuitive evolution of a single resonator based metasurface, other architectures are naturally possible with two or more resonance structures.

The coupled resonators of the unit cell has several key design parameters: slot resonator length, $\ell_{s}$ and width, $w_{s}$, ring

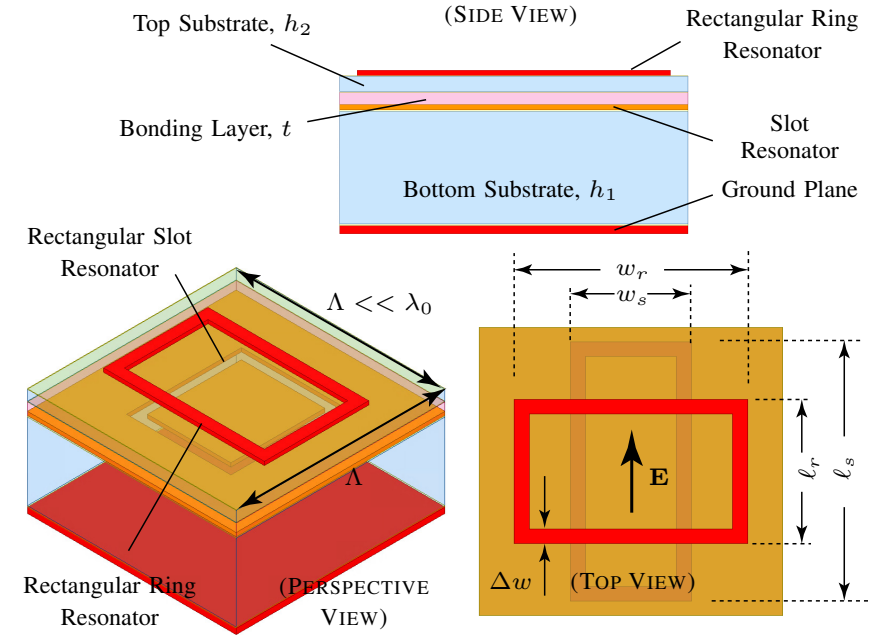

(a)

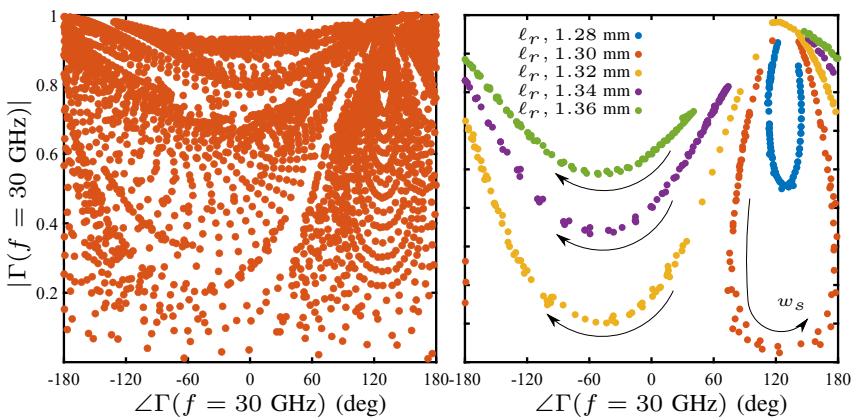

(b)

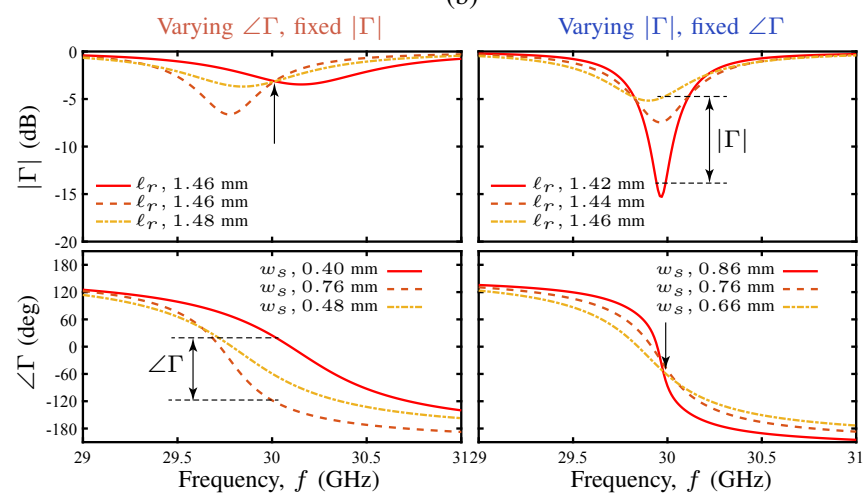

(c)

Fig. 2. The proposed unit cell configuration based on coupled resonators a) Detailed architecture showing the two physical resonators, material stack and properties. Simulation results (FEM-HFSS): b) full amplitude and phase coverage achievable at a fixed desired frequency of $30 \mathrm{GHz}$ and broadside excitation; achieved by varying various geometrical dimensions of the two resonators, along with specific sub-set where the ring, $\ell_{r}$ and slot length $w_{s}$ changed only while the slot and ring thicknesses are 110um, and $w_{r}=1.88 \mathrm{~mm}$ and $\ell_{s}=1.88 \mathrm{~mm}$. c) Specific examples of achieving a fixed reflection magnitude, but varying phase, and fixed phase but varying magnitude, where the slot and ring thicknesses are $110 \mathrm{um}$, and $w_{r}=1.88 \mathrm{~mm}$ and $\ell_{s}=1.88 \mathrm{~mm}$.

resonator length and width, $\ell_{r}$ and $w_{r}$, and the ring resonator thickness $\Delta w$, while the slot thicknesses always remains at a thickness of $110 \mu \mathrm{m}$, due to fabrication considerations. Fig. 2(b) [left] shows a typical simulated amplitude and phase coverage range when excited with normally incident planewaves, using FEM-HFSS, which are achievable using this unit cell by varying all these key design parameters for 
a fixed number of simulation runs and for a fixed design frequency $(30 \mathrm{GHz}$, here). Each point indicates that a set of resonator dimensions could be readily found, and that specific amplitude-phase pair is achievable. A near perfect amplitude coverage from full reflection to full absorption, and phase coverage of $2 \pi$ is observed, as desired.

It is further found that there is no simple relationship between the reflection magnitude and phase, with the five key parameters of the unit cell. Consequently, Fig. 2(b) essentially serves as the main look-up table of this unit cell for $30 \mathrm{GHz}$ operation frequency. A small subset of the full look-up table where all the parameters except $w_{s}$ and $\ell_{r}$, are kept constant is shown in the right of Fig. 2(b), which indicates some useful trends in the magnitude-phase coverage plot. Fig. 2(c) shows two examples to illustrate an independent magnitudephase combination response. The left figure shows specific configurations of the unit cell, where the magnitude is held constant at $30 \mathrm{GHz}$, while the reflection phase is varied. The right figure on the other hand, shows the reflection phase kept constant, but the magnitude varying from low to high reflection.

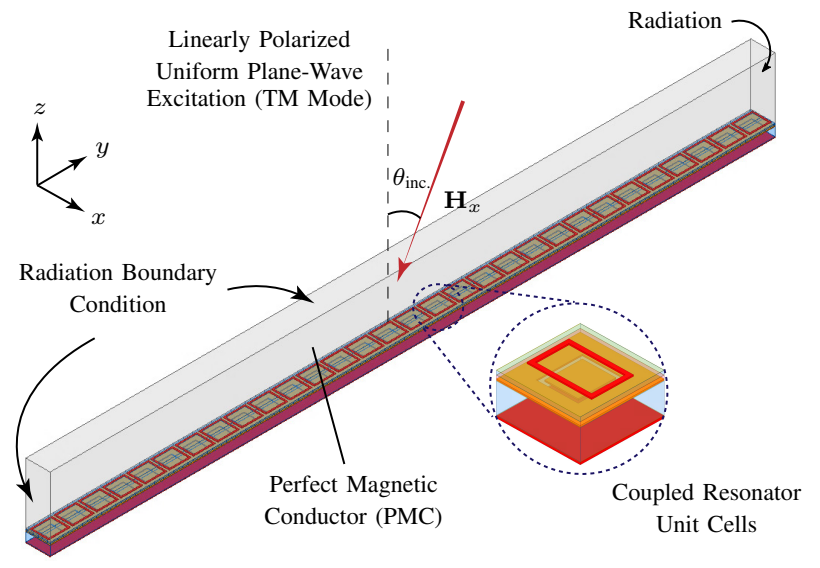

Fig. 3. A full-wave model built in Ansys FEM-HFSS showing the simulation setup of a 1-D unit cell array consisting of $N$ elments, forming the metasurface with various boundary conditions.

\section{B. Metasurface Design}

A metasurface can now be designed by cascading unit cells of Fig. 2(a) of varying resonator dimensions to realize a spatial complex reflectance along the metasurface to achieve a specified far-field reflection pattern. A full-wave model of the $N$ unit cell metasurface is shown in Fig. 3. The Metasurface is excited with a $y$-polarized uniform plane-wave, and perfect magnetic conductor (PMC) boundary condition is used along the $x$-direction to enforce uniformity along the $x$-axis. The unit cell geometry is varied along $y$, to engineer a desired complex reflectance profile, $\Gamma(y)$.

A simple way to obtain the space-dependent complex reflection profile is using standard antenna array theory, for instance, where the far-fields pattern is given by [32]

$$
E(\theta)=\sum_{n=1}^{N} a_{n} e^{j(n-1)\left\{k_{0} \Lambda \cos \theta\right\}}
$$

where $k_{0}$ is the free-space wave number at the design frequency $\omega, N$ is the number of unit cells in the metasurface and $a_{n}$ is the complex reflectance of the $n^{\text {th }}$ unit cell. Using standard antenna array synthesis, a simple estimation of the complex reflectance can be made for desired far-field patterns. For example, the complex reflectance $\left(a_{n}\right)$ can be calculated through a Chebyshev window [32]. The number of unit cells $(N)$ is provided to the function as well as the sidelobe magnitude factor which controls the beam width. This weighting factor is then multiplied by $e^{j n \beta}$ where $\beta$ represents the phase gradient across each unit cell and $n$ is the current unit cell number. This then produces $N$ complex weighting values $\left(a_{n}\right)$, from which the calculation for the far field is then determined through Eq. 2. The complex reflectance $\left(a_{n}\right)$ at each unit cell is the near field required along the surface, for each unit cell and is used to confirm and converge the solution to the desired near field and thus far field patterns.

Figure 4 shows few examples of the proposed metasurface reflector with various spatial profiles of complex reflectances and corresponding far-field reflection patterns. The first metasurface example is where an oblique incident plane wave at $\theta=-25^{\circ}$ is used, to generate an output reflection beam at an angle of $40^{\circ}$ at a design frequency of $30 \mathrm{GHz}$. Since the angle of reflection does not follow conventional Snell's law, a linear phase gradient $\angle \Gamma(y)$ is needed to steer the beam. Simultaneously, it is desired to change the reflection magnitude of the output beam to various levels, including almost a perfect absorption. Thus reflection magnitude control is required. Using (2), the complex reflectance is generated, and the look-up table of Fig. 2 is used to generate three different designs, exhibiting three different $|\Gamma|$ independently of the require phase gradient, as shown in the bottom plots of Fig. 4(a-c). The top plots of Fig. 4(a-c), show the fullwave simulated far-field reflection profile using the model of Fig. 3. As expected, an output reflection beam is successfully generated at $40^{\circ}$ in each case, with varying field magnitudes, as specified. Differences in the ideal reflectance profile and ones obtained from FEM-HFSS, may be attributed to interunit cells couplings in a non-uniform structure which are not accounted for in both the array factor of (2), as well as the look-up table of Fig. 2 obtained using ideal periodic boundary conditions.

A second example of a surface is a one that generates two reflection beams of specified characteristics when excited with a uniform plane-wave at an angle of $-10^{\circ}$. Two different designs are made. The first design generates a reflection beam in each half of the surface (i.e. one beam with anomalous reflection), where both the reflection angles do not follow conventional Snell's law, as shown in Fig. 3(d). A second design generates one anomalously reflected beam and another following conventional Snell's law, as shown in Fig. 3(e). In these examples, generally higher background reflection is observed compared to the specifications, which can be attributed to the noticeable differences in the near-field complex reflectance of the surface, specially for the phase. Nevertheless, in both cases, two reflection beams of specified beam characteristics are successfully generated. These two examples of asymmetric dual-beam surfaces, illustrates the usefulness of the proposed 

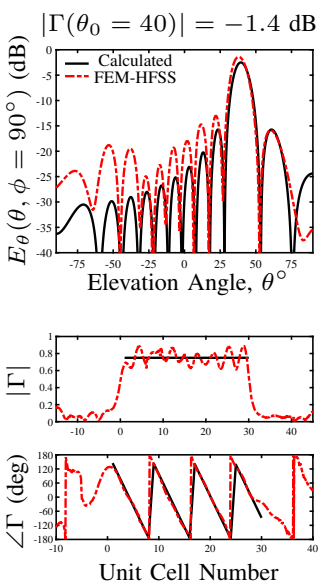

(a)
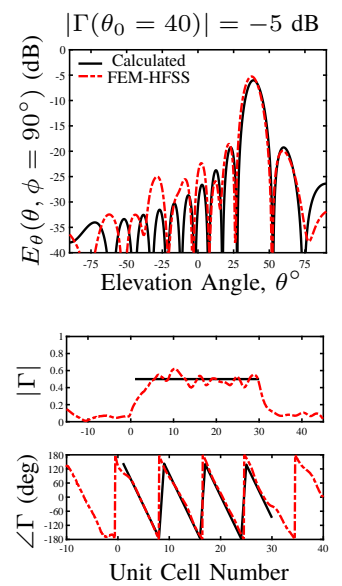

(b)
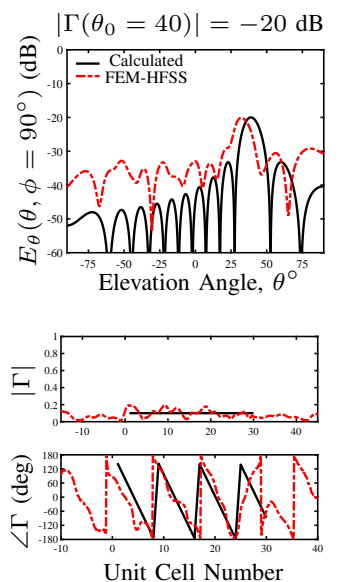

(c)
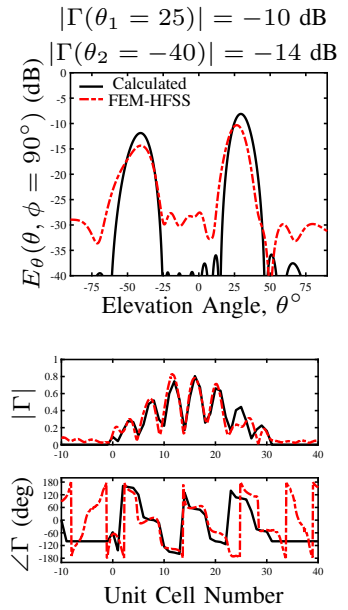

(d)
$\left|\Gamma\left(\theta_{1}=10\right)\right|=-10 \mathrm{~dB}$
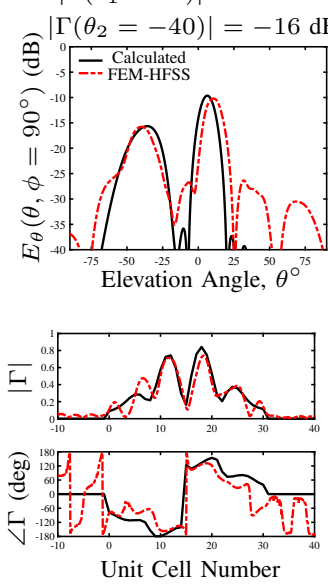

(e)

Fig. 4. Examples of multi-amplitude single beam and dual-beam metasurface reflector with specified placements of the two beams, showing there far-field reflection patterns and the corresponding near-field complex phase profiles. Various design specifications are provided in Tab. I.

TABLE I

Metasurface Design SumMary OF FEM-HFSS IN FIG. 4. $\theta_{\text {in }}(\mathrm{deg}) \quad \theta_{\text {out }}(\mathrm{deg}) \quad E_{\text {peak }}(\mathrm{dB}) \quad 3-\mathrm{dB}$ width, $\Delta \theta$

$\begin{array}{ccccc}\text { Single Beam \#1 } & -25 & 40 & -1.4 & 11 \\ \text { Single Beam \#2 } & -25 & 40 & -5 & 11 \\ \text { Single Beam \#3 } & -25 & 32 & -20 & 11 \\ \text { Dual Beam \#1 } & -10 & \{25,-40\} & \{-10,-14\} & \{12,18\} \\ \text { Dual Beam \#2 } & -10 & \{10,-40\} & \{-10,-16\} & \{11,16\}\end{array}$

coupled resonator metasurface reflector providing independent reflection amplitude and phase control, which are otherwise not possible to obtain using phase-only metasurfaces. The summary of the various beam characteristics is tabulated in Tab. I. It should be noted that the choice of these specifications were arbitrarily made to illustrate the concept.

\section{Measurement Results}

\section{A. Measurement Setup}

To measure the scattered field characteristics of various metasurface reflectors, no standard metasurface measurement setup exists. Consequently, an in-house measurement system is built that operates in the Ka-band (26.5-40 GHz), as shown in Fig. 5. It consists of a circular track on which a Tx and Rx standard gain horn antennas (Eravant/Sage Millimeter SAR2013-282F-E2, Ka-band horn with 20dBi gain) are installed and moved using an automated control. The two horn antennas are connected to the two ports of a vector network analyzer (PNA, Agilent N5230A). To enable co-angle measurements, where the Tx and Rx horns are located at the same angle (i.e. mono-static configuration), an angular tilt is added on each horn in the vertical plane in addition to slightly different heights, as shown in Fig. 5(c). The metasurface to be tested, is placed at the center of the circular track. The separation between the metasurface and the Tx/Rx horns is $700 \mathrm{~mm}$, to ensure far-field measurement conditions, which essentially limits the size of the surface in the given operation frequency band. Several absorbers were placed to minimize reflections from the background. A typical noise floor of $-30 \mathrm{~dB}$ is measured.

Next, to calibrate the system, a PEC reflector of the same size as the metasurface to be tested is first used, as a reference reflector. The measured angular field distribution and the peak field amplitude is then used to calibrate the system, which is then used to estimate the reflection characteristics

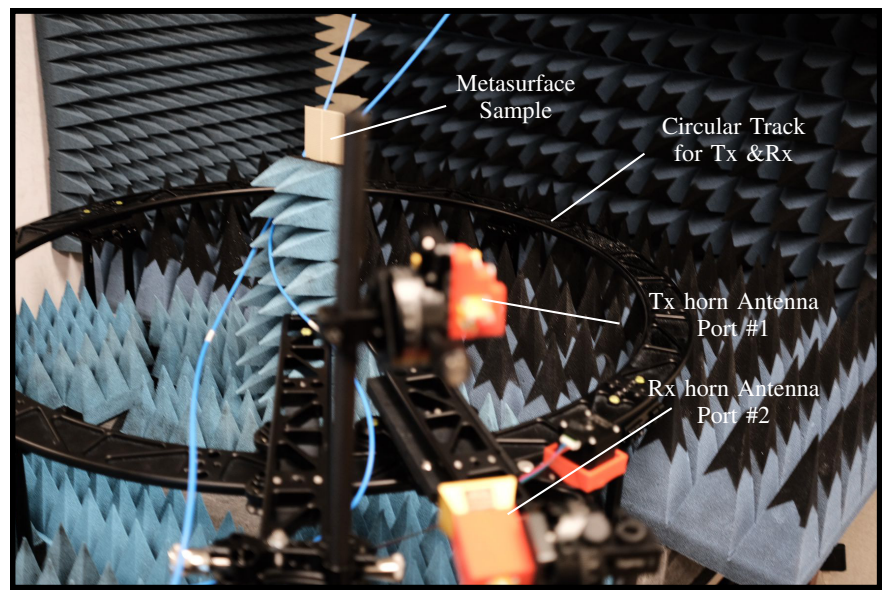

(a)

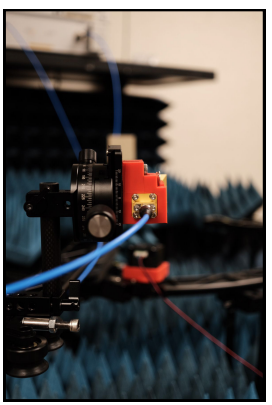

(b)

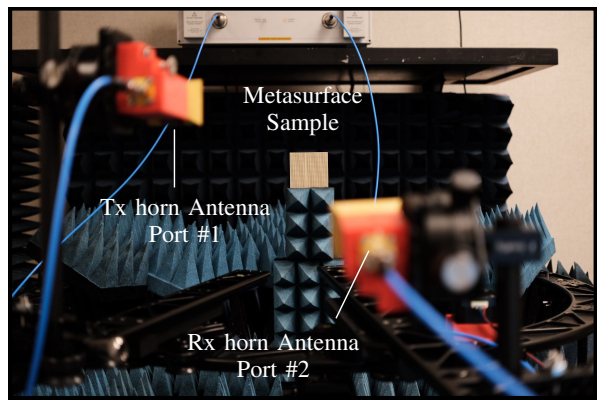

(c)
Fig. 5. Various photographs of the custom made metasurface measurement system operating in the Ka-band, showing the motorized transmitting and the receiving horn on a circular track, used to characterize various metasurface reflector prototypes. 
of the surface. The angular response of the PEC reflector at various frequencies was measured and compared with fullwave simulations (not shown here), and a typical amplitude difference is found to be $\approx 0.5 \mathrm{~dB}$ between the two. After the calibration step, the PEC reflector is replaced with a metasurface of interest for actual characterization.

\section{B. Metasurface Prototypes \& Results}

Next, all the metasurface designs of Tab. I operating at $30 \mathrm{GHz}$ were fabricated for testing based on the unit cell geometry of Fig. 2. The detailed fabrication parameters have already been summarized in Sec. II-A. Fig. 6 shows the results of the single beam steered metasurface with three different reflection amplitudes, where a picture of one of the prototypes is shown in Fig. 6(a) consisting of a $30 \times 30$ unit cell array. Two identical sets of each metasurface (\#1 and \#2) were fabricated to test the repeatability of the prototypes/measurements. The Tx horn is fixed at an angle of $\theta_{\text {inc. }}=-25^{\circ}$, while the $\mathrm{Rx}$ horn is moved around the metasurfaces in the front half space, i.e. $\theta \in\left\{-90^{\circ}, 90^{\circ}\right\}$. The top row shows the transmission frequency response $\left(S_{21}\right)$ of the surfaces at various $\mathrm{Rx}$ angular positions, for the cases of high reflection (large $S_{21}$ ), medium reflection and low reflection (small $S_{21}$ ), respectively. All the $S$-parameters plots are normalized with respect to the calibrated data measured using the reference PEC reflector. This broadband response helps to identify any frequency shifts that may have occurred due to fabrication and material tolerances. Indeed, a frequency downshift is observed of about $1 \mathrm{GHz}$, clearly evident from the location of the transmission dips across frequency. Next, angular field patterns were extracted at $29 \mathrm{GHz}$ for each case, and are shown in the bottom row of Fig. 6(b-d). A clear beam-tilting at $40^{\circ}$ is observed, specially for the high and medium reflection cases, while the transmission level is almost $-20 \mathrm{~dB}$ for the low reflection case approaching a strong absorption. The responses of two identical surfaces further show good repeatability of the measurements. In spite of a clear main reflection beam, in general the background reflection appears to be higher than expected, which could be due to spurious reflections from the environment, as the setup is not completely shielded in an enclosure. Nevertheless, the variation in the reflection amplitude, while the beam being steered, is clearly demonstrated with an acceptable agreement with the full-wave simulations.

The second set of measurements corresponds to the dual beam reflection patterns. The picture of one of the prototypes is shown in Fig. 7(a). The incident beam is fixed at $\theta_{\text {inc. }}=-10^{\circ}$, and two beams are generated to the left and right of the incident beam. Two different surfaces are fabricated with different beam specification, corresponding to the last two entries of Tab I, and their corresponding reflection responses are shown in Fig. 7(b-c). In both cases, a narrow but higher reflection beam is observed at $\theta_{2}$, as desired with very good agreement with full-wave simulations. The anomalously reflected beam on the left is lower in amplitude and wider in beamwidth. While noticeable ripples are observed on this beam, it has an acceptable agreement with the full-wave response in terms of the reflection amplitude and beam location, in particular. An improved measurement setup with better isolation from the environment is expected to reduce the background reflection and occurrence of local spurious reflections. Overall, both surfaces generated two distinct beams of desired characteristics, and the measurements for both single and double beam surfaces validate the proposed metasurface architecture to achieve independent amplitude and phase characteristics.

\section{CONCLUSions}

A static metasurface reflector based on a novel coupled resonator configuration has been proposed to independently control the reflection phase and magnitude of a linearly polarized incident fields, and has been demonstrated experimentally in the millimeter-wave Ka-band. The chosen practical design consists of rectangular ring coupled with a rectangular slot resonator backed by a grounded dielectric slab, due to its design simplicity and ease of implementation. By geometrically tuning various dimensions of the two resonators, a near-perfect amplitude-phase coverage has been achieved at a fixed design frequency of $30 \mathrm{GHz}$. Using the generated look-up table, various metasurface reflectors have been designed, simulated and successfully measured. To demonstrate the flexible beamforming capability of the proposed metasurface reflectors, illustrative examples of fixed beam steering with varying reflection magnitudes, and dual-beam pattern with specified reflection magnitude, reflection angles and beam-widths, has been demonstrated.

The proposed concept of utilizing coupled resonances to achieve independent reflection amplitude and phase, represents an attractive technique for achieving arbitrary complex reflection profiles from metasurfaces, without generating spurious cross-polarization. In addition, it does not require discrete resistive elements which are typically available with limited quantized values, thereby eliminating the lumped element assembly step in standard PCB fabrication and simplifying the overall prototyping process, making the proposed techniques ideal for high frequency implementations including various millimeter-wave bands. While a multi-layer unit cell architecture of the coupled ring and slot resonator has been shown here for demonstration due to its intuitive conceptualization, other resonant shapes are possible which may provide more optimized reflection response.

\section{ACKNOWLEDGEMENTS}

The authors acknowledge funding from the Department of National Defence's Innovation for Defence Excellence and Security (IDEaS) Program in support of this work.

\section{REFERENCES}

[1] C. Holloway, E. F. Kuester, J. Gordon, J. O’Hara, J. Booth, and D. Smith, "An overview of the theory and applications of metasurfaces: The two-dimensional equivalents of metamaterials," IEEE Antennas Propag. Mag., vol. 54, no. 2, pp. 10-35, April 2012.

[2] _ "An overview of the theory and applications of metasurfaces: The two-dimensional equivalents of metamaterials," IEEE Antennas Propag. Mag., vol. 54, no. 2, pp. 10-35, April 2012. 


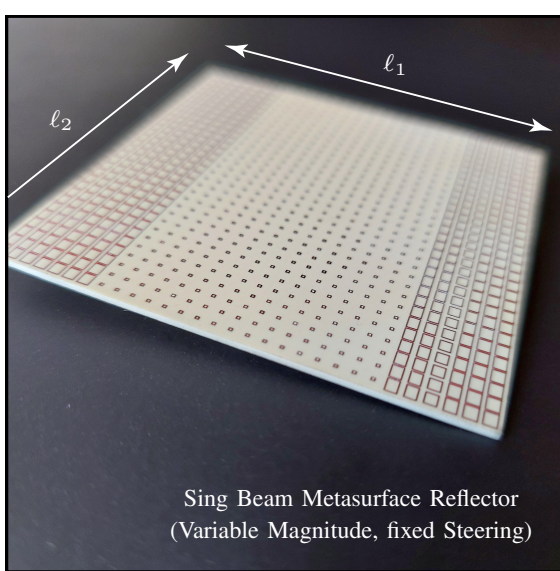

(a)
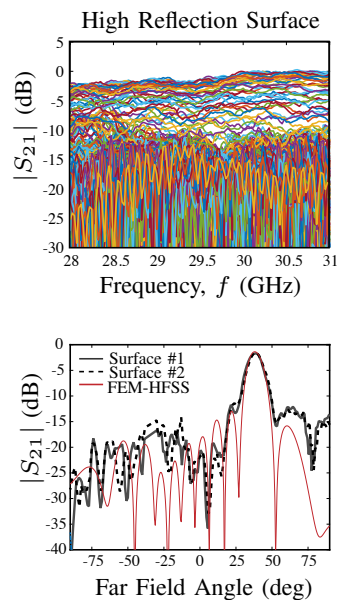

(b)
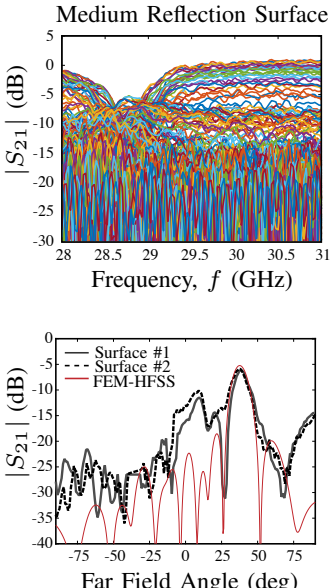

(c)
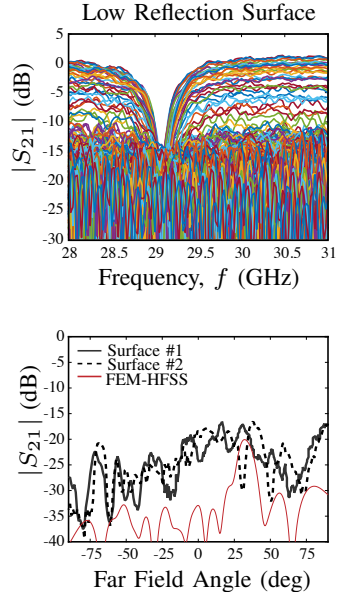

(d)

Fig. 6. A single beam metasurface reflector with varying magnitude and fixed steering angle of $40^{\circ}$ (constant phase gradient). a) Picture of one of the prototypes where $\ell_{1}$ and $\ell_{2}$ are $60 \mathrm{~mm}$. b-d) Frequency vs reflection where each line corresponds to an angle in the far field, showing varying level of reflection magnitude, along with far field patterns at $29 \mathrm{GHz}$. Metasurface is excited by a plane wave at $-25^{\circ}$.

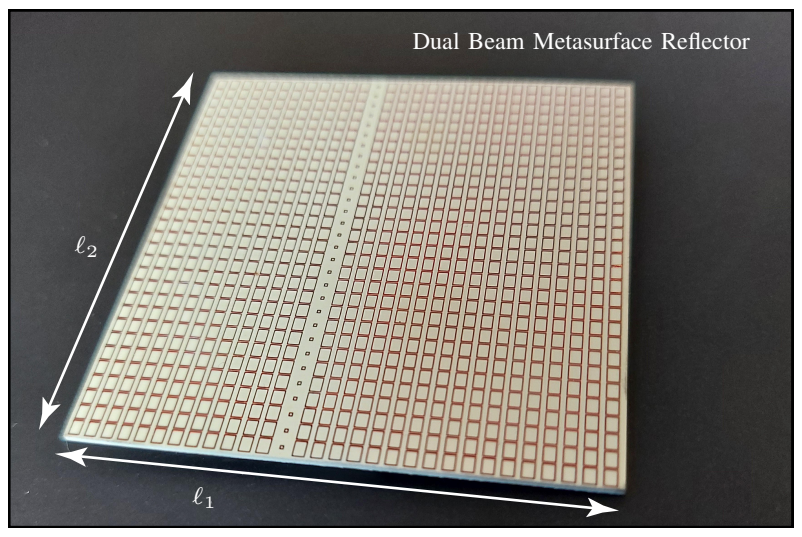

(a)

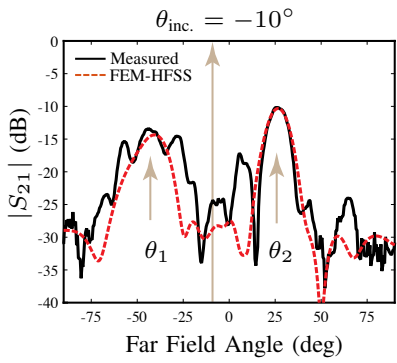

(b)

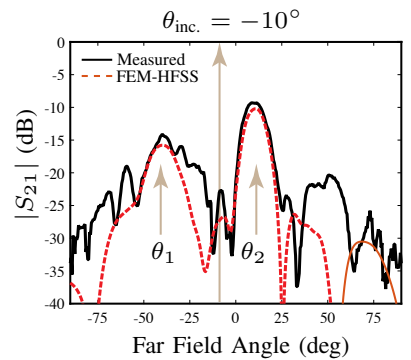

(c)
Fig. 7. Measured metasurface reflector producing two reflections beam at specified angles and with varying amplitude and beamwidths. a) A picture of one of the prototypes where $\ell_{1}$ and $\ell_{2}$ are $60 \mathrm{~mm}$. b) Far field pattern for dual beam at $29 \mathrm{GHz}$, with two beams reflected at $\theta_{2}=25^{\circ}$ at $-10 \mathrm{~dB}$ and $\theta_{1}=-40^{\circ}$ at $-14 \mathrm{~dB}$. c) Far field pattern for dual beam at $29 \mathrm{GHz}$ with two beams reflected at $\theta_{2}=10^{\circ}$ at $-10 \mathrm{~dB}$ and $\theta_{1}=-40^{\circ}$ at $-16 \mathrm{~dB}$. Input plane wave excitation at $-10^{\circ}$ in both cases.

[3] S. Chang, X. Guo, and X. Ni, "Optical metasurfaces: Progress and applications," Annual Review of Materials Research, vol. 48, no. 1, pp. 279-302, 2018.

[4] G. Oliveri, J. P. Turpin, J. A. Bossard, K. L. Morgan, D. H. Werner, and P. L. Werner, "Reconfigurable and tunable metamaterials: A review of the theory and applications," International Journal of Antennas and Propagation, vol. 2014, p. 429837, 2014.

[5] R. Fleury, F. Monticone, and A. Alù, "Invisibility and cloaking: Origins, present, and future perspectives," Phys. Rev. Applied, vol. 4, p. 037001 Sep 2015.

[6] H.-T. Chen, A. J. Taylor, and N. Yu, "A review of metasurfaces: physics and applications." Reports on progress in physics. Physical Society, vol. 79, no. 7, p. 076401, 2016.

[7] G.-Y. Lee, J. Sung, and B. Lee, "Recent advances in metasurface hologram technologies," ETRI Journal, vol. 41, no. 1, pp. 10-22, 2019.

[8] L. Huang, S. Zhang, and T. Zentgraf, "Metasurface holography: from fundamentals to applications," Nanophotonics, vol. 7, no. 6, pp. 11691190, 2018.

[9] X. Liu, F. Yang, M. Li, and S. Xu, "Generalized boundary conditions in surface electromagnetics: Fundamental theorems and surface characterizations," Appl. Sci., vol. 9, p. 1891, 2019.

[10] C. Pfeiffer and A. Grbic, "Metamaterial huygens' surfaces: Tailoring wave fronts with reflectionless sheets," Phys. Rev. Lett., vol. 110, p. 197401, May 2013.

[11] S. L. Jia, X. Wan, P. Su, Y. J. Zhao, and T. J. Cui, "Broadband metasurface for independent control of reflected amplitude and phase," AIP Advances, vol. 6, no. 4, p. 045024, 2016.

[12] H. Li, G. Wang, L. Zhu, X. Gao, and H. Hou, "Wideband beam-forming metasurface with simultaneous phase and amplitude modulation," Optics Communications, vol. 466, p. 124601, 2020. [Online]. Available: https://www.sciencedirect.com/science/article/pii/S0030401819308430

[13] H.-P. Li, G.-M. Wang, T. Cai, J.-G. Liang, and X.-J. Gao, "Phase- and amplitude-control metasurfaces for antenna main-lobe and sidelobe manipulations," IEEE Transactions on Antennas and Propagation, vol. 66, no. 10, pp. 5121-5129, 2018.

[14] Y. Yuan, S. Sun, Y. Chen, K. Zhang, X. Ding, B. Ratni, Q. Wu, S. N. Burokur, and C.-W. Qiu, "A fully phase-modulated metasurface as an energy-controllable circular polarization router," Advanced Science, vol. 7, no. 18, p. 2001437, 2020. [Online]. Available: https://onlinelibrary.wiley.com/doi/abs/10.1002/advs.202001437

[15] Y. Zhou, X. Cao, J. Gao, H. Yang, and S. Li, "Reconfigurable metasurface for multiple functions: magnitude, polarization and phase modulation," Opt. Express, vol. 26, no. 22, pp. 29451-29459, Oct 2018. [Online]. Available: http://www.opticsexpress.org/abstract.cfm? URI=oe-26-22-29451

[16] L. Liu, X. Zhang, M. Kenney, X. Su, N. Xu, C. Ouyang, Y. Shi, J. Han, W. Zhang, and S. Zhang, "Broadband metasurfaces with simultaneous control of phase and amplitude," Advanced Materials, vol. 26, no. 29, pp. 5031-5036, 2014. [Online]. Available: https://onlinelibrary.wiley.com/doi/abs/10.1002/adma.201401484

[17] Q. Wang, X. Zhang, Y. Xu, J. Gu, Y. Li, Z. Tian, R. Singh, S. Zhang, J. Han, and W. Zhang, "Broadband metasurface holograms: toward complete phase and amplitude engineering," Scientific Reports, vol. 6, no. 1, p. 32867, Sep 2016. [Online]. Available: https: //doi.org/10.1038/srep32867

[18] J. Ding, N. Xu, H. Ren, Y. Lin, W. Zhang, and H. Zhang, "Dual-wavelength terahertz metasurfaces with independent phase and 
amplitude control at each wavelength," Scientific Reports, vol. 6, no. 1, p. 34020, Sep 2016. [Online]. Available: https://doi.org/10.1038/srep34020

[19] M. Kim, A. M. H. Wong, and G. V. Eleftheriades, "Optical huygens' metasurfaces with independent control of the magnitude and phase of the local reflection coefficients," Phys. Rev. X, vol. 4, p. 041042, Dec 2014. [Online]. Available: https://link.aps.org/doi/10.1103/PhysRevX.4. 041042

[20] X. Zhang, Z. Tian, W. Yue, J. Gu, S. Zhang, J. Han, and W. Zhang, "Broadband terahertz wave deflection based on cshape complex metamaterials with phase discontinuities," Advanced Materials, vol. 25, no. 33, pp. 4567-4572, 2013. [Online]. Available: https://onlinelibrary.wiley.com/doi/abs/10.1002/adma.201204850

[21] S. Zhou, T. Li, and E. Li, "Design of resistor-loaded coding metasurface for independent amplitude and phase control," Journal of Electromagnetic Waves and Applications, vol. 0, no. 0, pp. 1-12, 2021.

[22] W. Che, H. Ye, Y. Xiong, Y. Chang, and C. Christopoulos, "A fast and efficient method for design of circuit analog absorbers consisting of resistive square loop arrays," in 2015 IEEE International Conference on Computational Electromagnetics, 2015, pp. 35-37.

[23] X. Q. Lin, P. Mei, P. C. Zhang, Z. Z. D. Chen, and Y. Fan, "Development of a resistor-loaded ultrawideband absorber with antenna reciprocity," IEEE Transactions on Antennas and Propagation, vol. 64, no. 11, pp 4910-4913, 2016.

[24] B. Zhu and Y. Feng, "Passive metasurface for reflectionless and arbitary control of electromagnetic wave transmission," IEEE Transactions on Antennas and Propagation, vol. 63, pp. 5500-5511, 2015.

[25] A. Z. Ashoor and S. Gupta, "Metasurface reflector with real-time independent magnitude and phase control," 2020.

[26] B. O. Zhu, K. Chen, N. Jia, L. Sun, J. Zhao, T. Jiang, and Y. Feng, "Dynamic control of electromagnetic wave propagation with the equivalent principle inspired tunable metasurface," Scientific Reports, vol. 4, May 2014.

[27] A. Leki and J. Beerten, "Generalized multiport representation of power systems using abcd parameters for harmonic stability analysis," Electric Power Systems Research, vol. 189, p. 106658, 2020. [Online]. Available: https://www.sciencedirect.com/science/article/pii/S0378779620304612

[28] R. J. Langley and E. A. Parker, "Equivalent circuit model for arrays of square loops," Electronics Letters, vol. 18, no. 7, pp. 294-296, 1982.

[29] Edwar and A. Munir, "Equivalent circuit analysis of square-loopresonator bpf with cross-shaped i/o coupling for $\mathrm{x}$-band frequency application," in 2017 International Conference on Control, Electronics, Renewable Energy and Communications (ICCREC), 2017, pp. 66-69.

[30] D. Ferreira, R. F. S. Caldeirinha, I. Cuias, and T. R. Fernandes, "Square loop and slot frequency selective surfaces study for equivalent circuit model optimization," IEEE Transactions on Antennas and Propagation, vol. 63, no. 9, pp. 3947-3955, 2015

[31] L. H. Weng, Y.-C. Guo, X.-W. Shi, and X.-Q. Chen, "An overview on defected ground structure," Progress In Electromagnetics Research B, vol. 7, no. 0, pp. 173-189, 2008

[32] C. A. Balanis, Advanced Engineering Electromagnetics. John Wiley and Sons, 1989. 